doi:10.1007/978-1-4419-6472-4_18 • www.stefancreemers.be • $₫$ info@stefancreemers.be

\title{
Modeling a hospital queueing network
}

\author{
Stefan Creemers \\ Marc Lambrecht
}

\begin{abstract}
Healthcare systems differ intrinsically from manufacturing systems. As such, they require a distinct modeling approach. In this article, we show how to construct a queueing network of a general class of healthcare systems. In order to analyze such networks, we use the parametric decomposition approach. Using this approach the network is decomposed into a set of single queueing systems which can be analyzed separately. Afterwards, results of these single queueing systems can be aggregated and general performance measures of the queueing network are obtained. In addition, we develop new expressions to assess the impact of service outages and use the queueing network to approximate patient flow times and to evaluate a number of practical applications.
\end{abstract}

\section{Introduction}

Whereas the origin of queueing theory dates back from the beginning of the previous century, networks of queues have only been studied for a few decades. The pioneering works of Jackson (1957 and 1963) showed that the stationary distribution of the number of customers in queue at a queueing network, is a product form of the stationary distributions at the individual workstations of the network. As a consequence, a queueing network can be decomposed into separate building blocks (i.e. the individual workstations) that can be analyzed separately to obtain the solution to the network as a whole. This approach is referred to as the parametric decomposition approach. The main advantage of the approach is that it enables the study of, otherwise intractable, complex queueing networks.

Unfortunately, the results obtained by Jackson (1957 and 1963) are only valid in socalled "Jackson networks" (i.e. queueing networks which assume Poisson arrival and service processes). When assuming a generalized queueing network (featuring general service and arrival processes), the product form solution no longer holds. As such, one requires another means to "link" the separate building blocks of the queueing network. This link is established in the form of a "linking equation". More specifically, a linking equation approximates the stochastic nature of the outgoing stream of customers at one of the workstations of the network. Using this information, we can assess the stochastic nature of the inflow of customers at the workstations further down the queueing network. As such, a linking equation literally "links" the results obtained at the separate workstations to obtain the solution of the network as a whole. Marshall (1968) was the first to study the stochastic nature of the outflow of customers at a queueing workstation. Ever since, a wide variety of linking equations (applicable to a wide variety of settings) has been developed. We refer the reader to Shanthikumar and Buzacott (1981), Buzacott and Shanthikumar (1985), Bitran and Tirupati (1988) and Suri, Sanders and Kamath (1993) for a nice review. 
Among others, these results have been extended and implemented in Whitt's Queueing Network Analyzer (1983), a powerfull tool that allows the analysis of a wide variety of complex queueing networks. Other noteworthy contributions to the domain of parametric decomposition of queueing networks include the works of Whitt (1994, 1995 and 1999a), Bitran and Tirupati (1988) and Lambrecht, Ivens and Vandaele (1998). A comprehensive overview of research on queueing networks in general and the parametric decomposition method in particular may be found with Askin (1993) and Hopp and Spearman (2000).

Queueing networks however, have mainly been studied in a manufacturing setting. Applications towards services in general and healthcare in particular are rarely seen. One of the reasons thereof is the difficulty of implementing the peculiarities of a service system into a methodology that is focussed on manufacturing systems. In what follows we discuss which problems may arise when modeling complex hospital queueing networks. Next we demonstrate how to use the parametric decomposition approach to model such queueing networks. In addition, we develop new expressions to assess the impact of service outages in a healthcare setting. The queueing network is used to test a variety of practical problems. More specifically, we demonstrate the impact on system performance resulting from the reduction of service outages and illustrate the beneficial effects of pooling. Moreover, we develop an optimization model that enables us to determine the optimal number of patients to be treated during a service session (e.g. a consultation time block). Finally we present some conclusions.

\section{Problem Description}

An important feature of healthcare processes (or services in general) is that the demand for resources is to a large extent unscheduled. As a consequence, there is a permanent mismatch between the demand for a treatment and the available capacity. Moreover, timely care is very important so interrupts are common in healthcare processes (the sense of urgency is almost always present). No wonder that healthcare is riddled with delays. No need to come up with a convincing example, we have all experienced that phenomenon. Delays are highly undesirable, not only from a psychological point of view (patient satisfaction) but also from an economic point of view. Government reimbursement systems are more and more based on a Justified Length of Stay (JLoS) system. DRG's (Diagnosis Related Groups) are characterized by a minimum and maximum length of stay (depending on parameters such as severity of the illness, age of the patient, ...). If a patient is dismissed before the JLoS is over, the hospital still collects a full reimbursement. On the other hand, if the patient remains in care for a period which exceeds the limit of the JLoS, the hospital has to pay for the extra costs involved. The JLoS of a DRG is determined in function of a national average length of stay. The system stimulates hospitals to continuously improve their performance. Moreover, improper scheduling and malfunctioning logistical systems cause lengths of stay that are too long. Insurance companies may reject reimbursement of these "denied days" because the delay is not medically necessary Hall, Belson, Muralli and Dessouky (2006). Delays also create a "hidden" hospital in analogy with the hidden company. In other words, such a hospital creates wasteful overhead.

Hall (2006) coined the term patient flow. It represents the ability of the healthcare sys- 
tem to serve patients quickly, reliably and efficiently as they move through stages of care. Queue and delay analysis can produce dramatic improvements in medical performance, patient satisfaction and cost efficiency of healthcare. Healthcare systems can be represented as a complex queueing network. The queueing models are helpful to determine the capacity levels (and the allocation of capacity) needed to respond to demands in a timely fashion (minimizing the delay). There is a demand side (the patient mix and the associated variability in the arrival stream) and a supply side (the hospital resources such as surgeons, nurses, operating rooms, waiting rooms, recovery, imaging machines, laboratories) in any healthcare process. Moreover, both demand and supply are inherently stochastic. This stochastic nature creates disturbances and outages during the process. It is the combination of capacity analysis and variability that makes queueing theory so attractive. The major objective is to identify factors influencing the flow time of patients, to identify levers of improvement and to analyze trade-offs. In this article we try to address some of the issues mentioned above.

Queueing models have been applied in numerous industrial settings and service industries. The number of applications in healthcare, however, is relatively small. This is probably due to a number of unique healthcare related features that make queueing problems particularly difficult to solve. In this section, we will review these features and where appropriate we will shortly discuss the methodological impact.

Before we dig into this issue, let's first discuss two important modeling issues in healthcare: the performance measures and the issue of pooled capacity.

The performance measures in healthcare systems focus on internal and external delays. The internal delay refers to the sojourn time of patients inside the hospital before treatment. The external delay refers to the phenomenon of waiting lists. Manufacturing systems may buffer with finished goods inventory, service systems rely more on time buffers and capacity buffers. Another important performance measure is related to the target occupancy (utilization) levels of resources. Average occupancy targets are often preferred by government and other institutional agents. Hereby, higher occupancy levels are preferred, but this results in longer delays. We are often confronted with conflicting objectives. Instead of determining capacity needs based on (target) occupancy levels, it is preferable to focus on delays. The key issue in delay has to do with the tail probability of the waiting time. The tail probability refers to the probability that a patient has to wait more than a specified time interval. Capacity needs (e.g. staffing) of an emergency department should be based on an upper bound on the fraction of patients who experience a delay of more than a specific time interval before receiving care from a physician (Green and Soares, 2007). The second modeling issue has to do with pooling. In general, pooling refers to the phenomenon that available inventory or capacity is shared among various sources of demand (well known examples are location pooling, commonality or flexible capacity). Pooling is based on the principle of aggregation and mostly comes down to the fact that we can handle uncertainty with less inventory or capacity. In healthcare systems, resources are usually dedicated to specific patient types, hospitals have separate units or departments by diagnostic type and bed flexibility is almost non-existing. As a result, pooling is absent. This explains the fact that most queueing models reported in the literature are dealing with parts of the hospital. Queueing models, however, can be used to model hospital wide systems and to evaluate the benefits of greater versus less specialization of care units or other resources (scanners, labs, ... ).

Let's now turn to a number of unique healthcare related features making queueing models 
in healthcare difficult to model and to solve.

Re-entry of patients and stochastic routings During consultation, patients may be routed to different facilities. The routing of a patient through hospital facilities is not deterministic. Instead, during the diagnosis stage there is a probabilistic routing. Moreover, patients require in many cases several consultations before surgery. Even after a patient is discharged from the hospital after surgery and recovery, the patient is subjected to a number of follow-up consultations. In other words, the queueing model must take care of re-entry of patients, creating additional work on top of the new patients. In most cases, the re-entry is correlated.

Service sessions for consultation and surgery In most queueing models time is considered as continuous and events are spread out over this continuous time scale. In services in general and in healthcare more specifically, resources are not continuously available. Instead, time is divided into "service sessions" for consultation (e.g. twice a week) or surgery (e.g. one day per week). Consequently we have to focus on service processes in which service takes place during predefined service sessions. Vacation models observe the queueing behavior of such systems in which servers are available during certain time intervals and are on "vacation" during the other time intervals.

Capacity related issues Hospitals operate within strict business restrictions. Resources are usually very scarce and consequently hospitals operate under high capacity utilization conditions. The so-called heavy traffic conditions are present. Heavy traffic conditions assume that all stations in the network are critically loaded. In such an environment, inaccurate results have a large impact on resulting performance measures.

Modeling of absences, disturbances and interruptions An important determinant of the flow time is variability. We distinguish two types of variability. Natural variability is variability that is inherent to the system process. Natural variability is much more substantial in healthcare as compared to manufacturing environments. Second, we have variability that can be related or assigned to a specific external cause. This variability is caused by unplanned absences of medical staff or interruptions during service operations. It is well known that variability induces waiting time. As a result the time available during consultation is often exceeded. This in turn is remedied by allowing overtime. Unfortunately, overtime modeling is a non-trivial issue in queueing.

\section{A hospital queueing system}

The features discussed in the previous section considerably complicate the modeling exercise. In order to demonstrate how to implement the features in a queueing model, we use an example hospital queueing system. The example concerns a typical hospital department involving consultation, surgery and recovery. The example we use throughout this paper is inspired by a real life case of the orthopedic department of the Middelheim hospital (Antwerp, 
Belgium) (Creemers and Lambrecht, 2007). We omit in this paper all practical data collection details of the case. We now and then provide numerical data to give the reader an idea of the problem dimension. In our example, the department employs six surgeons. Each of the surgeons is assigned a certain number of patients and no patient crossover between surgeons is assumed to take place. The base case deals in other words with the non-pooled capacity. Recovery occurs in an internal ward, an external ward or in the day hospital (depending on the disorder the patient is suffering from). In each of the wards 25 beds are reserved for patients of the hospital department under study. The capacity structure of the department is illustrated in Figure 1 .

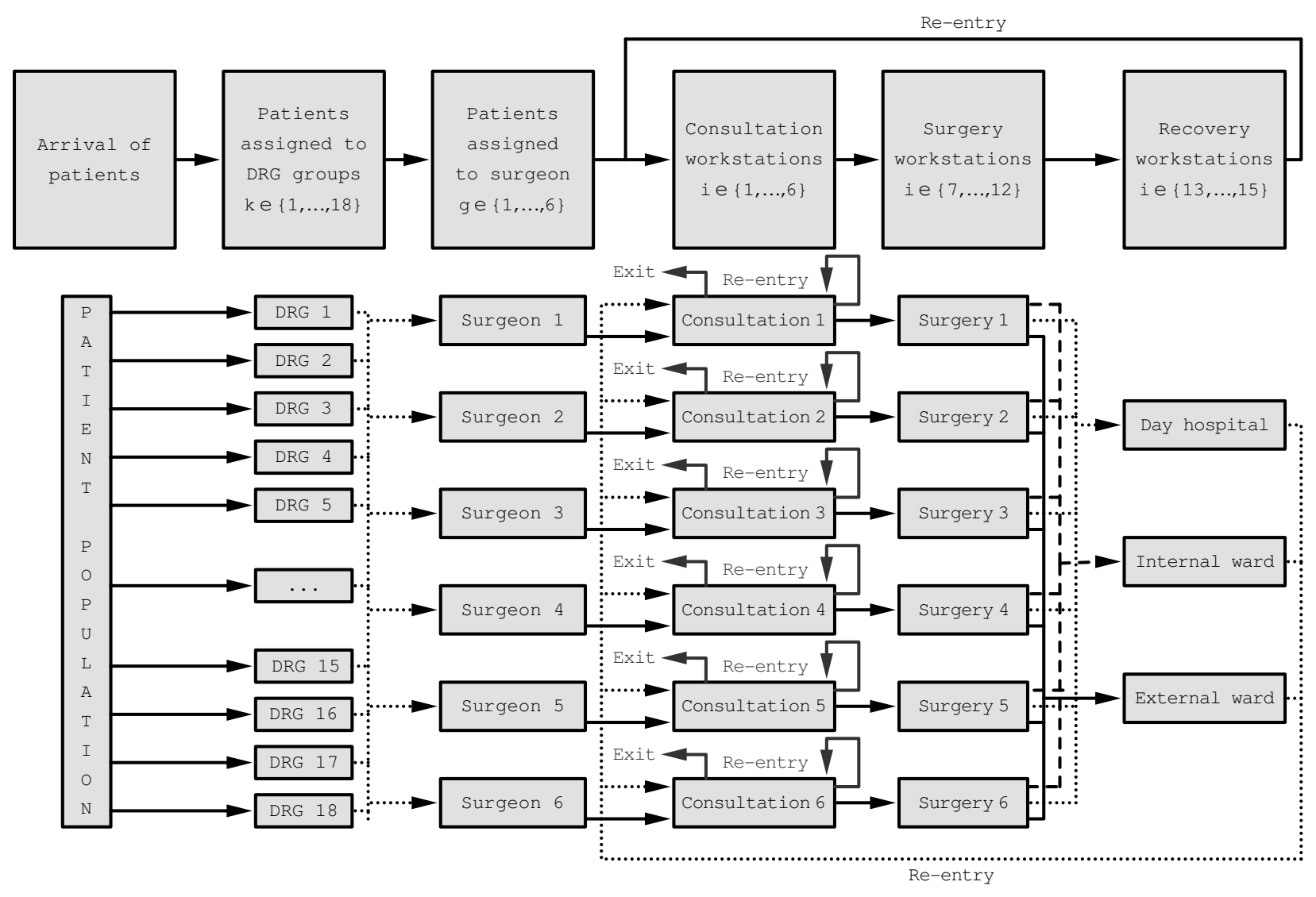

Figure 1: Hospital queueing network

Notwithstanding the fact that every patient is unique, we impose some general assumptions regarding the treatment process of a patient visiting the department. More specifically, we assume that every patient starts the treatment process with one or more consultations. Next, surgery is performed and a number of follow-up consultations is initiated. Finally the treatment process of a patient finishes and the patient leaves the hospital system. We assume that only elective surgery takes place and that the consultation process is appointment-based. Remark that it is possible to specify other patient routings (e.g. patients who refuse surgery, patients that do not longer need recovery, ...). In this example, however, we make use of a simple patient routing structure in order to preserve the transparency of the model.

With respect to the performance measures, we are interested in the total flow time of a 
patient at a workstation (i.e. consultation, surgery or recovery). We define the flow time as the total waiting time plus the processing time. With respect to the waiting time of a patient, a distinction is made between the internal waiting time and the external waiting time (Vissers, Bertrand and De Vries (2001) and Hall et al. (2006)). More specifically, the internal waiting time is the time spent inside the hospital prior to receiving service (at any of the workstations). The external waiting time is the time between the making of an appointment and the arrival of a patient at the hospital. The external waiting time can also be related to the "waiting list" phenomenon. As such, the total flow time of a patient consists of: (1) the external waiting time; (2) the internal waiting time; (3) the processing time. In the remainder of this text we will use $E[W]$ to denote the total flow time of a patient.

The data collection may be described in the following way (see also Figure 1). We start with a patient population (in our case we collected data on the consultation, surgery and recovery process of 3,300 patients) and divide it into groups of similar DRG's. We construct 18 DRG groups and use index $k, k \in\{1,2, \ldots, K\}$ for further identification (refer to Roth and Van Dierdonck (1995) and van Merode, Groothuis and Hasman (2004) for a detailed treatment on patient classification methodology). Next, the patients are assigned an individual surgeon (identified using index $g, g \in\{1,2, \ldots, G\}$ ). Surgeons as well as recovery wards may be considered as hospital resources. We use index $i, i \in\{1,2, \ldots, I\}$ to identify these resources. The surgeons perform both consultation $(i \in\{1,2, \ldots, 6\})$ as well as surgery $(i \in\{7,8, \ldots, 12\})$ tasks. Recovery takes place at the day hospital $(i=13)$, the internal ward $(i=14)$ or the external ward $(i=15)$.

In what follows we develop the queueing model. First we provide the mathematical derivations required to obtain the arrival and natural process times. Next, we adapt the model to include the effects of service outages, the availability of workstations and the characteristics of the aggregate arrival process.

\subsection{Modeling arrival rate and natural service times}

The queueing model of the hospital department may be presented as a network of $12 G / G / 1$ workstations (six surgeons performing both consultation and surgery) and $3 \mathrm{G} / \mathrm{G} / \mathrm{m}$ workstations (the recovery wards). The network is an open re-entry network with stochastic routings and is modeled using the principles of the parametric decomposition approach. While other approaches are available (e.g. Brownian motion queueing models), a previous study has shown that the parametric decomposition approach works best when modeling complex hospital systems (Creemers et al., 2007).

The queue discipline adhered to at each of the stations is FCFS. Any variation in the arrival of patients (e.g. the early, late, unannounced or not showing up of patients) is presumed to be absorbed in the variance of the arrival process. The model assumes infinite buffers to exist in front of every queue. Realizing that the buffers in front of the consultation and surgery workstation correspond to their respective waiting lists, it would be incorrect to restrain them in size. In real life, if patients contact the hospital to make an appointment for a consultation or a surgery, they will be issued an appointment date no matter how far ahead in time this date might be (i.e. we assume patients not to display any balkingor reneging-behavior when arriving or abiding at the queue). Hence buffer capacities are 
virtually unlimited. With respect to the recovery wards, one might argue that queue capacity is in fact limited. However, there are several reasons that are able to question this assertion. Next to rendering the model highly intractable, finite buffers do not necessarily correspond to reality since shortages of bed capacity at the wards are solved at the local level and in general do not prolong the sojourn time of a patient (this of course presumes the presence of unoccupied beds somewhere in the hospital). Therefore we will assume infinite buffers at all stages of the treatment process. Considering the multiclass re-entry environment of the queueing network, aggregation of the arrival and service process is required in order to perform a decomposition-based queueing analysis.

More formally, let $i(i \in\{1, \ldots, I\})$ denote the workstation in the network, let $k(k \in$ $\{1, \ldots, K\})$ denote the DRG group a patient belongs to and let $g(g \in\{1, \ldots, G\})$ denote the surgeon a patient is assigned to. As such, we have $K G$ classes of patients visiting a set of $I$ workstations. Let the pair $(k, g)$ denote the class of a patient (i.e. a patient of class $(k, g)$ is assigned a surgeon $g$ and belongs to DRG group $k$ ). Patients belonging to different classes are allowed to differ in terms of interarrival times, service times and routing. Assume interarrival times and service times of patients to be i.i.d. if they belong to one and the same class and assume them to be independently (but not necessarily identically) distributed otherwise. Let $\eta_{i(k, g)}$ denote the external arrival rate of a class $(k, g)$ patient at workstation $i$ (remark that external arrivals are only assumed to take place at the consultation workstations). The aggregate external arrival rate at a workstation $i$ equals:

$$
\eta_{i}=\sum_{k=1}^{K} \sum_{g=1}^{G} \eta_{i(k, g)}
$$

Note that expression 1 is a general expression, most of the time a workstation will be uniquely assigned to a single surgeon, making the summation over $g$ redundant.

We assume that the interarrival times of the external arrivals are exponentially distributed. Such an assumption poses only a slight restriction on the accuracy of the model while it has been shown by Palm (1943) and Khinchin (1960) that the sum of a large numbers of independent renewal processes (i.e. the arrival processes of the different classes of patients) will tend to a Poisson process. Considering the multitude of classes of patients, the approximation of the aggregate external arrival process by means of a Poisson process should be accurate. In addition, Lariviere and Van Mieghem (2004) showed that the assumption of exponential interarrival times is reasonable in many service systems.

Let $\gamma_{i(k, g)}$ denote the expected number of visits a class $(k, g)$ patient will make to workstation $i$ (remark that only the consultation workstations are assumed to be visited more than once). The aggregate arrival rate of patients at the consultation level equals:

$$
\lambda_{i}=\sum_{k=1}^{K} \sum_{g=1}^{G} \eta_{i(k, g)} \gamma_{i(k, g)}, \forall i \in\{1,2, \ldots, 6\} .
$$

Note that in contrast to the aggregate external arrival rate, which was assumed to be Poissondistributed, the aggregate arrival rate (at each of the workstations) is allowed to follow a general distribution. Further define the routing matrix $R$ in which the elements $r_{i j}$ indicate the probability of a patient to travel from station $i$ to station $j$ after service completion 
at station $i$. Adhering to standard conventions, we establish a node (of index $i=0$ ) from which external arrivals originate and which also serves as a sink for patients leaving the hospital system. Let $r_{i 0}$ indicate the probability of leaving the system when departing from station $i$. Conversely $r_{0 i}$ implies the probability of an external arrival occurring at station $i$. The probabilities $r_{i j}$ can be expressed as the the proportion of the arrivals at station $i$ that travel towards station $j$. When assuming the stability of the queueing network, the law of conservation of flows (what comes in, must go out) dictates:

$$
r_{i 0}=r_{0 i}=\frac{\eta_{i}}{\lambda_{i}} \forall i \in\{1,2, \ldots, 6\} .
$$

With respect to the surgery workstations, each patient visiting the hospital department is subjected to surgery exactly once. As such, one can infer that:

$$
\lambda_{i}=\eta_{i}, \forall i \in\{7,8, \ldots, 12\} .
$$

Hence the probability of transition from the consultation to the surgery level may be defined as:

$$
r_{i j}=\frac{\eta_{i}}{\lambda_{i}}, \forall i \in\{1,2, \ldots, 6\}, j=i+6 .
$$

Finally, at the consultation level, the probability of re-entry equals:

$$
r_{i i}=1-\left(r_{i 0}+r_{i j}\right)=1-\frac{2 \eta_{i}}{\lambda_{i}}, \forall i \in\{1,2, \ldots, 6\}, j=i+6 .
$$

The routing probabilities of transferring from a surgery workstation $i, i \in\{7,8, \ldots, 12\}$ towards a recovery ward $j, j \in\{13,14,15\}$ is obtained as follows:

$$
r_{i j}=\frac{\lambda_{j}^{(i)}}{\lambda_{i}}, \forall i \in\{7,8, \ldots, 12\}, \forall j \in\{13,14,15\},
$$

where $\lambda_{j}^{(i)}$ is the empirically observed arrival rate of patients at recovery workstation $j, j \in$ $\{13,14,15\}$ originating from surgery workstation $i, i \in\{7,8, \ldots, 12\}$. As such, the arrival rates at recovery equal:

$$
\lambda_{j}=\sum_{i=7}^{12} \lambda_{j}^{(i)}, \forall j \in\{13,14,15\} .
$$

From this we obtain:

$$
r_{i j}=\frac{\lambda_{i}^{(j+6)}}{\lambda_{i}}, \forall i \in\{13,14,15\}, \forall j \in\{1,2, \ldots, 6\} .
$$

All other routing probabilities stem directly from the structure of the model. A schematic summary of the routing matrix $R$ is presented in Table 1 . 
doi:10.1007/978-1-4419-6472-4_18 • www.stefancreemers.be • $₫$ info@stefancreemers.be

Table 1: Schematic summary of the routing matrix $R$

\begin{tabular}{c||c|c|c|c}
$i / j$ & 0 & $1-6$ & $7-12$ & $13-15$ \\
\hline \hline 0 & 0 & $\frac{\eta_{j}}{\lambda_{j}}$ & 0 & 0 \\
\hline $1-6$ & $\frac{\eta_{i}}{\lambda_{i}}$ & $\delta_{i j}\left(1-\frac{2 \eta_{i}}{\lambda_{i}}\right)$ & $\delta_{i j}\left(\frac{\eta_{i}}{\lambda_{i}}\right)$ & 0 \\
\hline $7-12$ & 0 & 0 & 0 & $\frac{\lambda_{j}^{(i)}}{\lambda_{i}}$ \\
\hline $13-15$ & 0 & $\frac{\lambda_{i}^{(j+6)}}{\lambda_{i}}$ & 0 & 0
\end{tabular}

Note that $\left(\delta_{i j}=1\right)$ if at least one of the patient classes travels from station $i$ to station $j$ and $\left(\delta_{i j}=0\right)$ otherwise.

Remark that other routing structures give rise to other routing probabilities. The routing structure and corresponding equations discussed in this section are only valid under the previously imposed assumptions concerning patient flow.

With respect to the service times, let $f_{i(k, g)}(x)$ denote the natural service time probability density function of a class $(k, g)$ patient visiting workstation $i$. Have $\frac{1}{\nu_{i(k, g)}}$ and $\sigma_{\nu_{i(k, g)}}^{2}$ represent the average natural service time for a class $(k, g)$ patient at workstation $i$ and its variance respectively. The natural process time excludes random interruptions, absences and any other external influence. Assume service times of different classes to be independent but not necessarily identically distributed. The probability that a randomly picked unit in front of the workstation is of class $(k, g)$ is given by $\frac{\lambda_{i(k, g)}}{\lambda_{i}}$, where $\lambda_{i(k, g)}$ is the total arrival rate of class $(k, g)$ patients at workstation $i$. Define the probability function of the aggregate natural service times at station $i$ as follows:

$$
f_{i}(x)=\sum_{k=1}^{K} \sum_{g=1}^{G} \frac{\lambda_{i(k, g)}}{\lambda_{i}} f_{i(k, g)}(x) .
$$

As a result the average natural service time requirement of a unit in front of the workstation amounts to:

$$
\frac{1}{\nu_{i}}=\sum_{k=1}^{K} \sum_{g=1}^{G} \frac{\lambda_{i(k, g)}}{\lambda_{i}} \frac{1}{\nu_{i(k, g)}} .
$$

When observing the variance of the aggregate natural service process, one can deduce that:

$$
\begin{aligned}
\sigma_{\nu_{i}}^{2} & =\sum_{k=1}^{K} \sum_{g=1}^{G} \frac{\lambda_{i(k, g)}}{\lambda_{i}} \int\left(x-\frac{1}{\nu_{i}}\right)^{2} f_{i(k, g)}(x) d x \\
& =-\frac{1}{\nu_{i}^{2}}+\sum_{k=1}^{K} \sum_{g=1}^{G} \frac{\lambda_{i(k, g)}}{\lambda_{i}}\left(\sigma_{\nu_{i(k, g)}}^{2}+\frac{1}{\nu_{i(k, g)}^{2}}\right) .
\end{aligned}
$$

We refer to $\sigma_{\nu_{i}}^{2}$ as a measure of the natural variability of the aggregate process times at workstation $i$. The same result was obtained by Whitt (1983) and has widely been adopted in literature (Whitt (1999b) and Haskose, Kingsman and Worthington (2002)). 


\subsection{Variability from preemptive and nonpreemptive outages}

With respect to service outages in healthcare, a large body of literature exists. Outages in a hospital setting have been the subject of discussion in Babes and Sarma (1991), Liu and Liu (1998a), Chisholm, Collison, Nelson and Cordell (2000) and Chisholm, Dornfeld, Nelson and Cordell (2001) among others. There is a consensus on the harmful effects of outages on patient flow times as well as on the quality of service. Outages result in congestion, unstable schedules and most importantly in overtime for staff members. We refer to Easton and Goodale (2005) for an excellent treatment of this issue. In this section, we focus on unplanned absences of medical staff and interruptions during service operations. Unplanned absences and interruptions during service activities have a major impact on flow times. Doctors and medical staff face various obligations which they have to attend to (making morning rounds, answering phones, patient check-ups, daily management, ...). In addition doctors often combine a hospital job and private consultation. These phenomena may cause a variable arrival pattern at the hospital (Liu et al., 1998a) and may lead to interruptions during the treatment process (Chisholm et al. (2000 and 2001) and Easton et al. (2005)). It is clear that hospital environments are characterized by substantial amounts of variability. As is argued in the literature (Hopp et al., 2000), variability induces waiting times. While in service industries variability cannot be countered by means of inventory in the traditional sense, patients will have to wait until capacity becomes available (Vissers et al. (2001), Vandaele and De Boeck (2003a) and Sethuraman and Tirupati (2005)). Besides the time buffer, hospitals often have to rely on a capacity buffer to mitigate the impact of variability and to maintain required service levels. In order to model service processes liable to outages, queueing theory proves to be an ideal tool. With respect to service outages and server unreliability, we face a vast amount of queueing literature. Surveys on the machine interference problem and server unreliability may be found in Stecke and Aronson (1985) and Haque and Armstrong (2007). Unreliable servers are often modeled using vacation models. Over the past decades, queueing systems with server vacations have received a lot of attention in the queueing literature. Vacation models observe the queueing behavior of systems in which the server begins a vacation (i.e. becomes unavailable) when certain conditions are met. For instance, imagine a doctor's office that has opening hours on Tuesday afternoons and on Friday evenings. On Tuesday, after service completion of the last patient, the doctor leaves on a "vacation" until Friday evening at which time service is resumed. At the end of service on Friday, a vacation is initiated until next Tuesday afternoon. We illustrate this process in Figure 2,

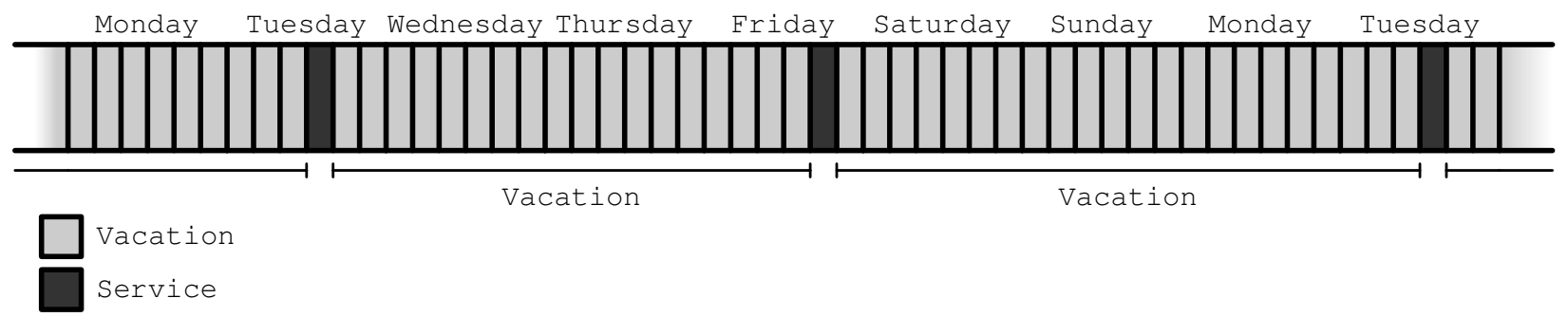

Figure 2: Illustration of a vacation model 
Next to the modeling of planned absences (e.g. a working schedule), vacation models may also be used to model unplanned server interruptions (e.g. a doctor who is called away for an emergency). A wide variety of vacation models exists. For a general overview, we refer to Doshi (1986), Takagi (1988) and Tian and Zhang (2006). In this work, however, we do not focus on vacation models. Instead, we consider an alternative, more intuitive approach to model service outages. This approach was first suggested by Hopp and Spearman (2000). In their work, Hopp and Spearman propose a transformation of the service process times to account for service outages. The results of Hopp and Spearman are widely accepted in the literature. In this work, we develop new expressions to model the impact of service outages that are peculiar to healthcare systems. In what follows, we first discuss the difference between preemptive and nonpreemptive outages. Next, we provide the means to model them.

\subsubsection{Outages, classification and impact}

As was indicated previously, the service process of a patient may be interrupted or postponed. These outages will increase the natural service times. We call these increased, adjusted service times, effective processing times. It is the total time "seen" or "experienced" by a patient at a workstation. The effective process time random variable is of primary interest to determine flow times.

We distinguish between preemptive and nonpreemptive outages. Preemptive and nonpreemptive outages will impact the service process and will give rise to increased levels of traffic intensity (resulting in the so-called effective utilization rate or effective traffic intensity).

Let us first discuss the nonpreemptive outages. Nonpreemptive outages typically occur between jobs, rather than during jobs. They occur at the beginning of each service session (i.e. at the start of a consultation work shift) whenever a doctor or another member of the medical staff is absent (e.g. due to late arrival). We may refer to such an outage as unplanned absences and define the mean and variance of the amount of time absent as $\frac{1}{\mu_{s}}$ and $\sigma_{s}^{2}$ respectively (i.e. absence times are allowed to follow a general distribution). Furthermore we assume an average number of patients (represented by $n$ ) to arrive in between two consecutive absences. This is an important feature of the model. Indeed, $n$ may be considered as the number of patients in a service session (e.g. a consultation work shift). Each start of a service session may induce a delay due to an absence. In other words, the number of patients in a service session is a decision variable and is comparable to a lot sizing decision. Evaluating different service session sizes (i.e. different values of $n$ ) may provide key managerial insights. We will address this issue in an upcoming section.

Next to nonpreemptive outages, we also allow for preemptive outages to take place. Preemptive outages occur whenever a doctor is interrupted during a consultation activity. These interruptions will be modeled in an approach which builds on the tradition set by Hopp and Spearman (2000). They are characterized by a Mean Time To Interrupt $\left(\tau_{f}\right)$ and a Mean Time To Resolve $\left(\tau_{r}\right)$. The model presented in Hopp and Spearman (2000) presumes interrupts to occur only during actual service time. However, in a hospital setting it is not inconceivable that interrupts take place during the resolve time induced by a previous interrupt as well. For instance, if the service process of a patient is interrupted by a phone call, it is still possible for a doctor to be called away for an emergency, to receive another 
call, ....

In what follows, we present the main results on nonpreemptive as well as preemptive outages. In a final subsection, we present results on the joint occurrence of nonpreemptive and preemptive outages. In order to maintain transparency of the model and of notation, we impose the following assumptions: (1) service outages only occur at the consultation level (i.e. only workstations $i, i \in\{1,2, \ldots, 6\}$ are affected); (2) for each of the surgeons, the impact of outages is identical (i.e. $\frac{1}{\mu_{s}}, \sigma_{s}^{2}, n, \tau_{f}$ and $\tau_{r}$ remain the same for each of the workstations at the consultation level).

\subsubsection{Nonpreemptive outages}

We define a nonpreemptive outage to occur whenever the succession of two events is based on the number of services performed in between (hence, setups, rework, maintenance, .... are all extensions that are able to capitalize on the technique discussed in this section). Applied to our setting, we have that $n$ patients are treated (on average) in between two consecutive absence possibilities. Assume that the length of services and absence times does not depend on the service history (i.e. they are independent of prior services and absence times). The absence times themselves are distributed following a probability density function $f_{s}(x)$. The average absence time and its variance are represented by $\frac{1}{\mu_{s}}$ and $\sigma_{s}^{2}$. The service time of the $n^{\text {th }}$ patient includes part service time, part absent time. We refer to the service time of the $n^{\text {th }}$ patient as the combined service time. We illustrate these concepts in Figure 3 .

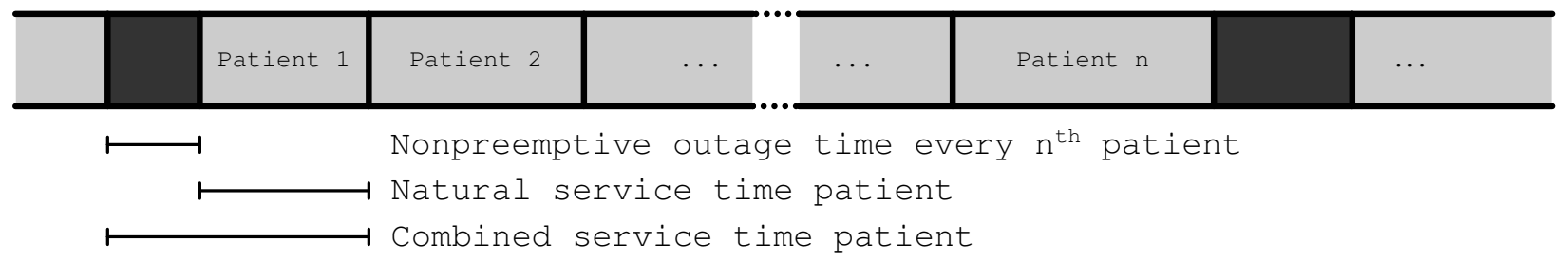

Natural service time

$\square$ Nonpreemptive outage time

Figure 3: The combined service time

One can consider the services that are preceded by an absent period as a separate class of patients that has a probability $\frac{1}{n}$ of randomly being picked in front of the workstation. The other services as a whole have a probability $\left(\frac{(n-1)}{n}\right)$ of randomly being picked. Therefore, we can define the mean aggregate service times including the effect of absence times as follows:

$$
\begin{aligned}
\frac{1}{v_{i}}= & {\left[\left(\frac{n-1}{n}\right) \sum_{k=1}^{K} \sum_{g=1}^{G} \frac{\lambda_{i(k, g)}}{\lambda_{i}} \int f_{i(k, g)}(x) x d x\right]+} \\
& {\left[\frac{1}{n} \sum_{k=1}^{K} \sum_{g=1}^{G} \frac{\lambda_{i(k, g)}}{\lambda_{i}} \iint f_{i(k, g)}(x) f_{s}(y)(x+y) d y d x\right], } \\
= & \frac{1}{\nu_{i}}+\frac{1}{n \mu_{s}} .
\end{aligned}
$$


With respect to the variance of the aggregate service time (including absence times) at the consultation workstations we develop the following expression:

$$
\begin{aligned}
\sigma_{v_{i}}^{2}= & {\left[\left(\frac{n-1}{n}\right) \sum_{k=1}^{K} \sum_{g=1}^{G} \frac{\lambda_{i(k, g)}}{\lambda_{i}} \int f_{i(k, g)}(x)\left(x-\frac{1}{v_{i}}\right)^{2} d x\right]+} \\
& {\left[\frac{1}{n} \sum_{k=1}^{K} \sum_{g=1}^{G} \frac{\lambda_{i(k, g)}}{\lambda_{i}} \iint f_{i(k, g)}(x) f_{s}(y)\left(x+y-\frac{1}{v_{i}}\right)^{2} d y d x\right], } \\
= & \sigma_{\nu_{i}}^{2}+\frac{\sigma_{s}^{2}}{n}+\frac{1}{\mu_{s}^{2}}\left(\frac{n-1}{n^{2}}\right) .
\end{aligned}
$$

The above expression is equivalent to that of Hopp and Spearman (2000) and is valid under the assumption that the combined service times as well as ordinary service times are independently distributed.

\subsubsection{Preemptive outages}

We refer to service interruptions as preemptive outages. Doctors being called away on emergencies, answering phone calls, ... are typical examples. The average time between two consecutive interrupts is defined as $\tau_{f}$ whereas $\tau_{r}$ refers to the average time it takes to resolve an interruption. Preemptive outages prove to be more difficult to model while they occur after the elapsing of a variable amount of time (i.e. a mean time to interrupt $\tau_{f}$ ), rather than after a number of patients being processed. Under the assumption that the time between two consecutive interrupts is exponentially distributed, expressions for mean and variance have been obtained. With respect to preemptive outages, we make a distinction between two different scenarios. On the one hand, one might presume preemptive outages to occur only during actual service time. As such preemptive outages do not take place during the resolve times induced by previous outages. Remark that this does not imply that the service process of a single patient cannot be interrupted more than once. On the other hand, one might assume preemptive outages to occur during resolve times as well (e.g. as indicated previously, doctors may be be interrupted when already engaged in resolving a previous interrupt). While this latter instance can be seen as an extension of the former, we will first discuss outages occurring exclusively during actual service time. Define $\tau_{r_{0_{j}}}$ as the resolve time of the $j^{\text {th }}$ preemptive outage that occurred during the service process of one and the same patient. The mean and variance of the resolve times are given by $\tau_{r}$ and $\sigma_{r}^{2}$. In addition, resolve times of different outages are assumed to be i.i.d.. The service process of a patient thus faces the probability of encompassing several interrupts that prolong its service duration. The service time of a patient (including interrupts) at a workstation $i$ can be expressed as:

$$
\frac{1}{\omega_{i}}=\frac{1}{\nu_{i}}+\sum_{j=1}^{J_{0}} \tau_{r_{0_{j}}} .
$$

As such, the random variable $\frac{1}{\omega_{i}}$ incorporates both the natural service time $\frac{1}{\nu_{i}}$ as well as the resolve times of interrupts that occurred during service. Moreover, $J_{0}$ denotes the number of preemptive outages that occurred during the service process of a unit. $J_{0}$ is a random variable that follows a Poisson distribution (i.e. we assume the time between two consecutive 
interrupts to be exponentially distributed) and its mean and variance both equal $\left(\frac{1}{\left(\nu_{i} \tau_{f}\right)}\right)$. We face a sum of random variables (the resolve times $\tau_{r_{0_{j}}}$ ) in which the number of random variables (the number of interrupts $J_{0}$ ), is a random variable itself. Assume that $J_{0}$ and $\tau_{r_{0_{j}}}$ $(\forall j \in \mathbb{N})$ are i.i.d. variables. In addition assume the mean as well as the variance of $\tau_{r_{0}}$ to be equal for all $j \in \mathbb{N}$. Therefore, the mean and variance of the sum of $J_{i_{0}}$ random variables $\tau_{r_{0_{j}}}$ can be expressed as (Dudewicz and Mishra, 1988):

$$
\begin{gathered}
E\left[S_{0}\right]=E\left[J_{0}\right] E\left[\tau_{r_{0_{j}}}\right] \\
\sigma_{S_{0}}^{2}=E\left[J_{0}\right] \sigma_{r}^{2}+E\left[\tau_{r_{0_{j}}}\right]^{2} \sigma_{J_{0}}^{2},
\end{gathered}
$$

where $S_{0}$ is the random variable representing the sum of $J_{0}$ resolve times $\tau_{r_{j}}$. In other words we have that:

$$
S_{0}=\sum_{j=1}^{J_{0}} \tau_{r_{0_{j}}}
$$

The mean and variance of the sum of resolve times can be defined as:

$$
\begin{gathered}
E\left[S_{0}\right]=\frac{1}{\nu_{i}} \frac{\tau_{r}}{\tau_{f}}, \\
\sigma_{S_{0}}^{2}=\frac{1}{\nu_{i}} \frac{\sigma_{r}^{2}+\tau_{r}^{2}}{\tau_{f}} .
\end{gathered}
$$

The mean aggregate service time including the effect of interrupts may be expressed as:

$$
E\left[\frac{1}{\omega_{i}}\right]=\frac{1}{\nu_{i}} \frac{\tau_{f}+\tau_{r}}{\tau_{f}}
$$

This corresponds to the expression presented in Hopp and Spearman (2000) in which the natural service time is divided by an availability factor in order to incorporate the effect of interrupts. Next we have a look at the variance of the service times including the effect of preemptive outages during service time. We start with the approximation of the second moment:

$$
E\left[\left(\frac{1}{\omega_{i}}\right)^{2}\right]=\left(\sigma_{\nu_{i}}^{2}+\frac{1}{\nu_{i}^{2}}\right)\left(1+\frac{\tau_{r}}{\tau_{f}}\right)^{2}+\sigma_{S_{0}}^{2} .
$$

Using the expression for the second moment we obtain the variance of the service times including the effect of interrupts:

$$
\sigma_{\omega_{i}}^{2}=\sigma_{\nu_{i}}^{2}\left(1+\frac{\tau_{r}}{\tau_{f}}\right)^{2}+\sigma_{S_{0}}^{2} .
$$

This expression once more matches the formula derived in Hopp and Spearman (2000). The above expressions hold if and only if the Poisson-distributed preemptive outages take place during service itself. In what follows, we relax this assumption and allow for interrupts to take place during the resolve times induced by previous interrupts. 
In order to approach this problem, we divide the interrupts into different sets. Let $l$ $(l \in \mathbb{N})$ denote the set index. We define $\tau_{r_{l_{j}}}$ to be the resolve time of the $j^{\text {th }}$ interrupt belonging to the set of index $l$ (i.e. the interrupt is said to be of order $l$ ). Without loss of generality assume that interrupts of order 0 occurred during actual service, interrupts of order 1 occurred during the resolve times of interrupts of order $0, \ldots$ In general, interrupts of order $l$ took place during the resolving of interrupts of order $(l-1)$. Figure 4 provides further insight.

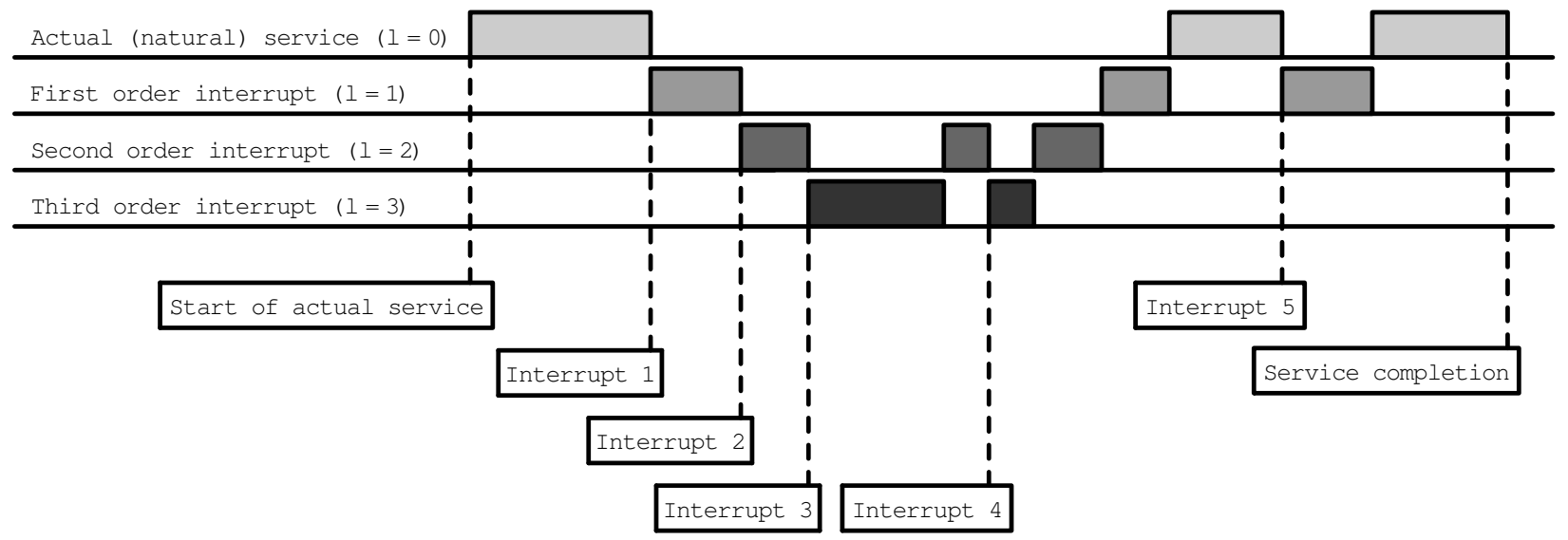

Figure 4: Interrupted service process of a single patient

In addition define $S_{l}$ as the sum of resolve times corresponding to interrupts of order $l$. We have that:

$$
S_{l}=\sum_{j=0}^{J_{l}} \tau_{r_{l_{j}}},
$$

where $J_{l}$ is the number of interrupts belonging to the set of index $l$. $J_{l}$ follows a Poisson distribution and its mean and variance equal:

$$
E\left[J_{l}\right]=\sigma_{J_{l}}^{2}=\frac{1}{\nu_{i} \tau_{f}}\left(\frac{\tau_{r}}{\tau_{f}}\right)^{l} .
$$

One can infer that:

$$
\begin{gathered}
E\left[S_{l}\right]=\frac{\tau_{r}}{\nu_{i} \tau_{f}}\left(\frac{\tau_{r}}{\tau_{f}}\right)^{l}, \\
\sigma_{S_{l}}^{2}=\frac{1}{\nu_{i} \tau_{f}}\left(\frac{\tau_{r}}{\tau_{f}}\right)^{l}\left(\sigma_{r}^{2}+\tau_{r}^{2}\right) .
\end{gathered}
$$

Using the same reasoning applied previously, one can express the mean aggregate service time including the effect of all order interrupts as follows:

$$
E\left[\frac{1}{\omega_{i}}\right]=\frac{1}{\nu_{i}} \frac{\tau_{f}}{\tau_{f}-\tau_{r}} .
$$


Using these parameters, the second moment is expressed as:

$$
E\left[\left(\frac{1}{\omega_{i}}\right)^{2}\right]=\left(\sigma_{\nu_{i}}^{2}+\frac{1}{\nu_{i}^{2}}\right)\left[1+2 \frac{\tau_{r}}{\tau_{f}-\tau_{r}}+\left(\frac{\tau_{r}}{\tau_{f}-\tau_{r}}\right)^{2}\right]+\frac{1}{\nu_{i}} \frac{\sigma_{r}^{2}+\tau_{r}^{2}}{\tau_{f}-\tau_{r}}
$$

As a result, the variance of the service time at a workstation $i$ (including the impact of all order interrupts) is given by:

$$
\sigma_{\omega_{i}}^{2}=\frac{\tau_{f}^{2} \sigma_{\nu_{i}}^{2}+\frac{1}{\nu_{i}}\left(\tau_{f}-\tau_{r}\right)\left(\sigma_{r}^{2}+\tau_{r}^{2}\right)}{\left(\tau_{f}-\tau_{r}\right)^{2}}
$$

\subsubsection{Combining preemptive and nonpreemptive outages}

In many hospital settings, both preemptive and nonpreemptive outages may surface. While it is impossible to interrupt the service process in the instance of a nonpreemptive outage (e.g. a doctor who arrives late), we only consider the case in which both types of outages cannot occur simultaneously. The average service time incorporating this combined effect at a workstation $i$ can be expressed as:

$$
\begin{aligned}
\frac{1}{\psi_{i}}= & {\left[\left(\frac{n-1}{n}\right) \sum_{k=1}^{K} \sum_{g=1}^{G} \frac{\lambda_{i(k, g)}}{\lambda_{i}} \int f_{i_{f(k, g)}}(x) x d x\right]+} \\
& {\left[\frac{1}{n} \sum_{k=1}^{K} \sum_{g=1}^{G} \frac{\lambda_{i(k, g)}}{\lambda_{i}} \iint f_{i_{f(k, g)}}(x) f_{s}(y)(x+y) d y d x\right], } \\
= & E\left[\frac{1}{\omega_{i}}\right]+\frac{1}{n \mu_{s}},
\end{aligned}
$$

where $f_{i_{f(k, g)}}(x)$ is the probability density function of consultation service times of a class $(k, g)$ patient at a workstation $i$ including the effect of all order interrupts. Its mean and variance are given by $E\left[\frac{1}{\omega_{i}}\right]$ and $\sigma_{\omega_{i}}^{2}$ respectively. We refer to $\frac{1}{\psi_{i}}$ as the effective service time while it equals the service time experienced by the patient (and as such includes the impact of outages). The variance of the effective service times at a workstation $i$ may be expressed as:

$$
\begin{aligned}
\sigma_{\psi_{i}}^{2}= & {\left[\left(\frac{n-1}{n}\right) \sum_{k=1}^{K} \sum_{g=1}^{G} \frac{\lambda_{i(k, g)}}{\lambda_{i}} \int f_{i_{f(k, g)}}(x)\left(x-\frac{1}{\psi_{i}}\right)^{2} d x\right]+} \\
& {\left[\frac{1}{n} \sum_{k=1}^{K} \sum_{g=1}^{G} \frac{\lambda_{i(k, g)}}{\lambda_{i}} \iint f_{i_{f(k, g)}}(x) f_{s}(y)\left(x+y-\frac{1}{\psi_{i}}\right)^{2} d y d x\right], } \\
= & \sigma_{\omega_{i}}^{2}+\frac{\sigma_{s}^{2}}{n}+\frac{1}{\mu_{s}^{2}}\left(\frac{n-1}{n^{2}}\right) .
\end{aligned}
$$

These results allow us to take service outages into account when assessing hospital performance measures.

\subsubsection{Including the time availability of workstations}

It is well known that many services do not operate continuously over time. Consultation and surgery typically operate during certain time intervals (service sessions) which means that only a proportion of the total available time can be used effectively. Vacation models are often 
applied to solve this problem. Another way to handle the problem is to rescale all service processing times so that they fit a preset uniform time scale. In this study we agreed on a 24 hours per day, 7 days per week time scale (basically because this is the appropriate time scale for recovery processes). Let $A_{i}$ denote the availability of workstation $i ; A_{i}$ represents the available time in proportion to the preset uniform time scale. For instance, if a workstation operates only 6 hours per day, then the availability equals $25 \%$.

When rescaling the service times established in the previous sections, we obtain the total effective service times:

$$
\begin{aligned}
\frac{1}{\mu_{i}} & =\frac{1}{A_{i} \psi_{i}}, \forall i \in\{1,2, \ldots, 6\}, \\
\frac{1}{\mu_{i}} & =\frac{1}{A_{i} \nu_{i}} \forall i \in\{7,8, \ldots, 15\}, \\
\sigma_{i}^{2} & =\frac{\sigma_{\psi_{i}}^{2}}{A_{i}^{2}}, \forall i \in\{1,2, \ldots, 6\}, \\
\sigma_{i}^{2} & =\frac{\sigma_{\nu_{i}}^{2}}{A_{i}^{2}} \forall i \in\{7,8, \ldots, 15\} .
\end{aligned}
$$

The above procedure results in the total effective service times including natural process time, the effect of outages and the impact of availability of workstations. The mean total effective service time and its variance can now be used to compute the squared coefficient of variation of the service times:

$$
C_{s_{i}}^{2}=\sigma_{i}^{2} \mu_{i}^{2}
$$

\subsubsection{Squared coefficient of variation of the aggregate arrival process}

In order to approximate the parameters of the aggregate arrival process, some more challenging arithmetics are needed. It was pointed out by Albin (1984) that if at least one of the interarrival time distributions, constituting the arrival process, does not stem from a Poisson process, the resulting aggregate interarrival times do no longer hold the property of independence. As a result the analytical analysis of the aggregate arrival process becomes highly intractable. Therefore approximations will be adopted to assess the variance and, more important, the squared coefficient of variation of the aggregate arrival process. The squared coefficients of variation of the aggregate arrivals at the different workstations will be extracted using a technique which was pioneered by Shanthikumar and Buzacott (1981). This technique implies the use of a set of linear equations which has to be solved in order to obtain the squared coefficients of variation of the arrivals. This approach is widely adopted in literature (Askin, 1993) and was later generalized by Lambrecht et al. (1998). Using the technique that was outlined in Lambrecht et al. (1998), we are given a set of $I$ equations:

$$
-\sum_{i=1}^{I} \lambda_{i} r_{i j}^{2}\left(1-\rho_{i}^{2}\right) C_{a_{i}}^{2}+\lambda_{j} C_{a_{j}}^{2}=\sum_{i=1}^{I} \lambda_{i} r_{i j}\left(r_{i j} \rho_{i}^{2} C_{s_{i}}^{2}+1-r_{i j}\right)+\eta_{j} C_{a_{\eta_{j}}}^{2}
$$

where $\eta_{j}$ and $C_{a_{\eta_{j}}}^{2}$ denote the rate and squared coefficient of variation of the aggregate external arrival process at station $j$ respectively. In addition, $\rho_{i}$ represents the effective 
traffic intensity at workstation $i$ and equals $\frac{\lambda_{i}}{\mu_{i}}$. While all elements except the $I$ squared coefficients of variation are known, we are presented with a system of $I$ equations yielding $I$ unknowns. Solving this set of linear equations provides us with the $I$ unknown squared coefficients of variation (i.e. $C_{a_{i}}^{2} ; \forall i \in\{1, \ldots, I\}$ ).

With all model parameters firmly defined, we now have a solid base to carry out the performance evaluation of the hospital department. In the upcoming section we discuss a numerical example of the model presented above and provide some practical applications.

\section{Applications}

In this section, we discuss a numerical example using the queueing model described in the previous section. Next, we illustrate the devastating impact of service interruptions on patient flow times. Subsequently, we show the potential gains obtained by pooling hospital resources. Finally, we present an optimization model to determine the optimal number of patients to be treated during a service session.

\subsection{Numerical example}

The numerical example presented in this section builds on data gathered at the orthopedic department of the Middelheim hospital in Antwerpen (Belgium). Using these empirical data as inputs, the flow time of patients at the hospital department may be assessed using socalled flow time expressions. A variety of flow time expressions are available in the queueing literature. A previous study has shown the Kingman equation to yield accurate results when assessing the flow times of patients in complex hospital systems (Creemers et al., 2007). As such, in the remainder of this article, we will use the Kingman equation to determine patient flow times. With respect to the Kingman equation, one can define the expected flow time of a patient at workstation $i$ as follows (Hopp et al., 2000):

$$
E\left[W_{i}\right]=\left(\frac{C_{a_{i}}^{2}+C_{s_{i}}^{2}}{2}\right)\left(\frac{\rho_{i}^{\sqrt{2\left(m_{i}+1\right)}-1}}{m_{i}\left(1-\rho_{i}\right)}\right) \frac{1}{\mu_{i}}+\frac{1}{\mu_{i}},
$$

where $m_{i}$ denotes the number of parallel servers at workstation $i\left(m_{i}=25 \forall i \in\{13,14,15\}\right)$. If only a single server is present (i.e. at workstations $i, i \in\{1,2, \ldots, 12\}$ ), no pooling is assumed to take place and the formula reduces to (Kingman, 1962):

$$
E\left[W_{i}\right]=\left(\frac{C_{a_{i}}^{2}+C_{s_{i}}^{2}}{2}\right)\left(\frac{\rho_{i}}{1-\rho_{i}}\right) \frac{1}{\mu_{i}}+\frac{1}{\mu_{i}} .
$$

Using the empirical data, resulting flow times at each of the workstations are obtained. The results are presented in Table 2 and Table 3 (all results are expressed in minutes unless indicated otherwise). While no waiting occurs at the wards (i.e. the process of recovery takes place immediately after surgery) the performance measures of workstations 13 to 15 are not included here. 
Table 2: Summary Table of the model results (workstations 1 to 6 )

\begin{tabular}{lcccccc}
\hline$i$ & 1 & 2 & 3 & 4 & 5 & 6 \\
\hline$\frac{1}{\psi_{i}}$ & 24.85 & 24.85 & 24.85 & 24.85 & 24.85 & 24.85 \\
$\frac{1}{\mu_{i}}$ & 310.7 & 690.4 & 310.7 & 167.9 & 155.3 & 248.5 \\
$C_{s_{i}}^{2}$ & 1.334 & 1.334 & 1.334 & 1.334 & 1.334 & 1.334 \\
$\frac{1}{\lambda_{i}}$ & 329.8 & 741.5 & 317.0 & 174.5 & 167.5 & 268.8 \\
$C_{a_{i}}^{2}$ & 1.026 & 1.418 & 1.051 & 0.759 & 0.752 & 0.952 \\
$A_{i}$ & 0.080 & 0.036 & 0.080 & 0.148 & 0.160 & 0.100 \\
$\rho_{i}$ & 0.942 & 0.931 & 0.980 & 0.962 & 0.927 & 0.925 \\
$E\left[W_{i}\right]$ (days) & 4.360 & 9.402 & 12.90 & 3.219 & 1.547 & 2.593 \\
\hline
\end{tabular}

Table 3: Summary Table of the model results (workstations 7 to 12)

\begin{tabular}{lcccccc}
\hline$i$ & 7 & 8 & 9 & 10 & 11 & 12 \\
\hline$\frac{1}{\nu_{i}}$ & 110.0 & 96.20 & 89.17 & 57.50 & 56.35 & 93.18 \\
$\frac{1}{\mu_{i}}$ & 1048 & 2004 & 1351 & 845.7 & 593.2 & 1035 \\
$C_{s_{i}}^{2}$ & 0.266 & 0.406 & 0.203 & 0.171 & 0.165 & 0.274 \\
$\frac{1}{\lambda_{i}}$ & 1,111 & 2,111 & 1,380 & 883.4 & 620.5 & 1,073 \\
$C_{a_{i}}^{2}$ & 1.089 & 1.121 & 1.074 & 1.058 & 1.068 & 1.070 \\
$A_{i}$ & 0.105 & 0.048 & 0.066 & 0.068 & 0.095 & 0.090 \\
$\rho_{i}$ & 0.943 & 0.950 & 0.979 & 0.957 & 0.956 & 0.965 \\
$E\left[W_{i}\right]$ (days) & 8.907 & 21.38 & 29.42 & 8.674 & 5.918 & 14.14 \\
\hline
\end{tabular}

With respect to consultation, no distinction was made between the different surgeons. One can observe that the effective service time (including the effect of interrupts and absences) amounts to 24.85 minutes (the natural service time amounting to 15 minutes). The coefficient of variation equals 1.334 (the natural coefficient of variation amounting to 0.6386). Arrival rates and their variances depend on the number of patients visiting each surgeon. The utilization rates of the surgeons are all very high, which translates into significant patient flow times varying from 1.5 days to 12.9 days.

Similar observations may be made with respect to surgery. Here we allow surgeons to have different processing times depending on the type of surgery they perform. In addition, observe the significantly longer waiting times for patients at the surgery level.

\subsection{The impact of interrupts}

The impact of interrupts on medical practice has been observed by Harvey, Jarrett and Peltekian (1994), Lehaney, Clarke and Paul (1999), Chisholm et al. (2001), France, Levin, Hemphill, Chen, Rickard, Makowski, Jones and Aronsky (2005), Volpp and Grande (2006), 
Table 4: Impact of interrupts (expressed in minutes) on patient flow time (expressed in days) at a single workstation

\begin{tabular}{ccccccccc}
\hline$\tau_{f}$ & $E[W]$ & $\rho$ & $\tau_{f}$ & $E[W]$ & $\rho$ & $\tau_{f}$ & $E[W]$ & $\rho$ \\
\hline 10.4 & 183.2 & 0.998 & 11.6 & 16.24 & 0.984 & 18 & 4.433 & 0.943 \\
10.5 & 93.58 & 0.997 & 11.8 & 14.35 & 0.982 & 20 & 3.393 & 0.936 \\
10.6 & 63.28 & 0.995 & $\mathbf{1 2 . 0}$ & $\mathbf{1 2 . 9 0}$ & $\mathbf{0 . 9 8 0}$ & 25 & 3.288 & 0.924 \\
10.7 & 48.05 & 0.994 & 12.5 & 10.43 & 0.975 & 30 & 2.968 & 0.916 \\
10.8 & 38.88 & 0.993 & 13.0 & 8.880 & 0.971 & 40 & 2.652 & 0.907 \\
10.9 & 32.76 & 0.992 & 14.0 & 7.029 & 0.963 & 60 & 2.401 & 0.897 \\
11.0 & 28.38 & 0.990 & 15.0 & 5.966 & 0.957 & 80 & 2.294 & 0.893 \\
11.2 & 22.54 & 0.988 & 16.0 & 5.276 & 0.952 & & & \\
11.4 & 18.82 & 0.986 & 17.0 & 4.791 & 0.947 & & & \\
\hline
\end{tabular}

Tucker and Spear (2006) and Gabow, Karkhanis, Knight, Dixon, Eiser and Albert (2006) among others. All agree on the detrimental effects of interrupts on patient flow time. In order to demonstrate these detrimental effects, we present a number of scenarios in which we gradually reduce the impact of interrupts. We build on the setting of the hospital department discussed previously. To maintain transparency, we focus on a single consultation workstation (i.e. the only workstations that are susceptible to interrupts during the service process). We adjust the mean time to interrupt (i.e. $\tau_{f}$ ) at this workstation to assess the varying impact of interrupts (all other model parameters remain unchanged). The results are given in Table 4. Note that we used the third workstation to study the impact of various degrees of interrupts (the results corresponding to the numerical example are indicated in bold). Figure 5 illustrates the phenomenon graphically. 


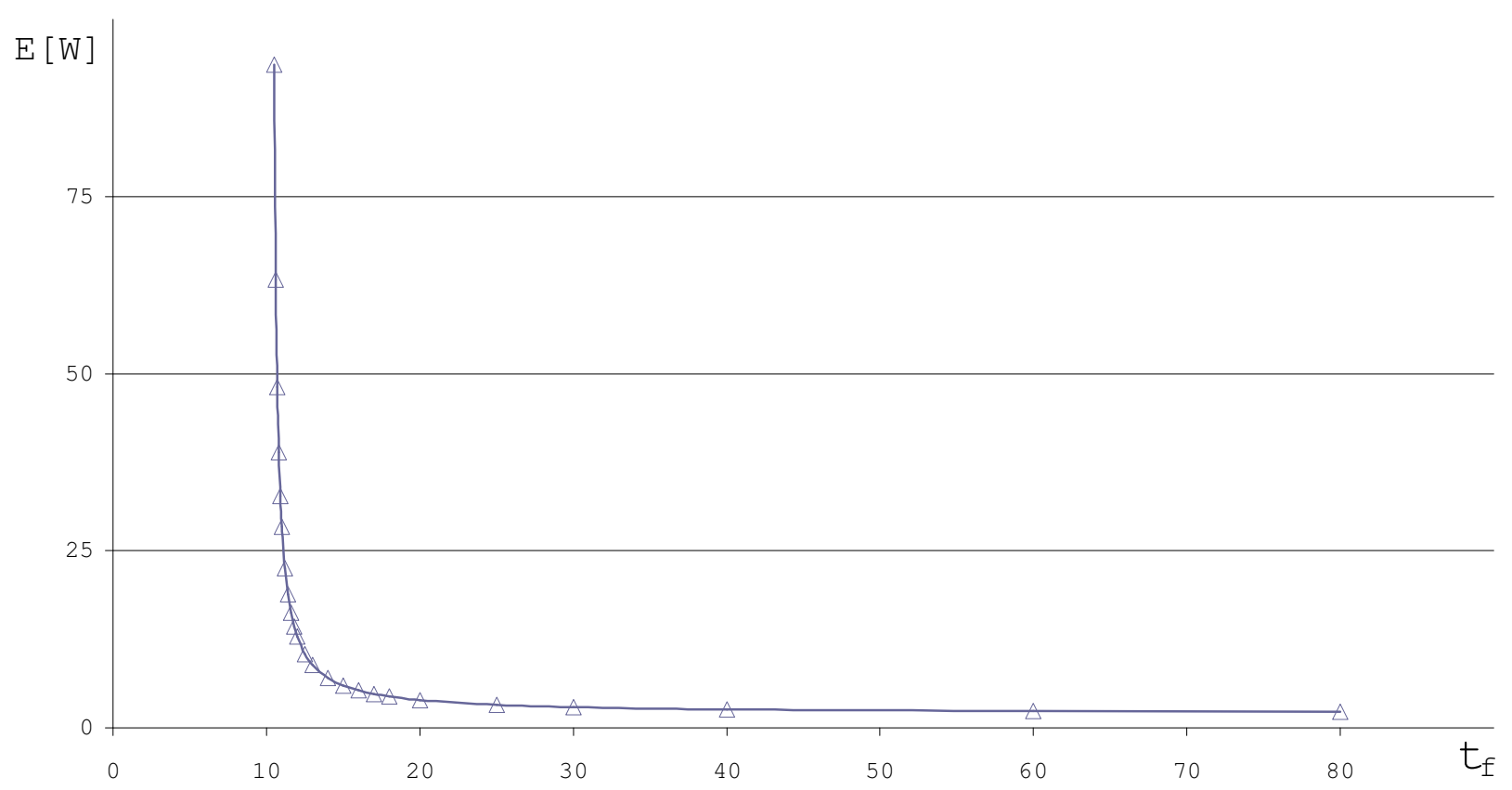

Figure 5: Varying impact of interrupts (expressed in minutes) and the effect on patient waiting times (expressed in days)

It is clear that heavy traffic systems (i.e. systems which operate under high workload) benefit greatly from even a small reduction in utilization rate. Unfortunately, only limited means are available to achieve such a reduction in utilization rate. A variety of options arise:

- The most obvious way to reduce the effective utilization is process improvement. Continuous improvement and six sigma programs are very beneficial. Reducing the frequency of interrupts can be classified in this category.

- Expand capacity; hospital resources such as operating theatres, scanners and other equipment are often operating at maximum capacity. Expanding capacity would be an effective means to reduce hospital workload. However, expanding capacity is often very expensive or is simply impossible (e.g. due to legal constraints).

- Limit patient volumes; a reduction in hospital workload might also be achieved by limiting the amount of patients receiving treatment. Pursuing this option however, results in loss of hospital income and a reduced level of service.

In the literature, valuable insights are provided that offer guidance in the quest to reduce the impact of interrupts. For instance, Harvey et al. (1994) suggest the pooling of paging of doctors (next to telephone calls, paging calls are one of the largest sources of interrupts) in order to decrease variability in individual paging patterns. France et al. (2005) propose the use of information systems (e.g. an electronic whiteboard) and team training to enhance performance. Tucker et al. (2006) suggest the redesign of treatment processes (e.g. outsourcing of administrative tasks) in order to make service more robust against preemptive outages. 
In addition Tucker et al. (2006) and Volpp et al. (2006) propose the filtering of non-urgent communication towards medical staff. These and other practical guidelines enable hospital decision makers to minimize the impact of interrupts on the service process.

\subsection{The impact of pooling}

Pooling refers to the aggregation (consolidation) of the demand from multiple items into one, such that the consolidated demand can be satisfied from a single buffer. More specifically, capacity pooling refers to the idea of sharing available capacity among various sources of demand (e.g. patient classes). In a hospital setting this refers to the sharing of expensive diagnostic equipment, wards or labs. In a non-pooling environment, each resource fulfills its own demand, relying solely on its own capacity. In a pooled environment, demand is aggregated and fulfilled from a single shared facility. A rich literature on pooling in queueing systems exists. For an excellent overview, we refer to Benjaafar and Cooper (2005) and Yu and Benjaafar (2006).

It has long been known that pooling is beneficial to system performance. More specifically, pooling allows to maintain a specified level of service quality (e.g. patient flow times) with less capacity requirements. The beneficial effect of pooling stems from the increased ability of the system to cope with variability. For instance, in pooled systems, it is much less likely for the queue to be empty. As such, the impact of variability in the arrival pattern of patients (patients may arrive early, late or may even fail to show up at all) or in the service process of surgeons is minimized.

In this section, we demonstrate the impact of server pooling by means of a small experiment. We build on the setting of the hospital department discussed in the previous sections. In the experiment the servers at the consultation and surgery level are pooled. The following assumptions are imposed:

- Patients are treated by the first surgeon available for service, even if the patient does not belong to the patient population corresponding to that surgeon.

- Surgeon working schedules are identical and no structural constraints are imposed (i.e. it should be possible to service 6 patients simultaneously).

Returning to our example setting, the six consultation and the six surgery workstations are replaced by a single consultation and a single surgery workstation respectively. Each of these workstations has six parallel servers in operation. The resulting queueing network contains five workstations $i, i \in\{1,2, \ldots, 5\}$. Let station 1 to 5 represent consultation, surgery, day hospital, internal ward and external ward respectively. When retaining all other characteristics of the setting discussed in the previous sections, one can use the multiserver Kingman equation to obtain patient flow times. The resulting performance measures are presented in Table 5 (the non-pooled flow times are the weighted average of the flow times observed at the consultation and surgery workstations presented in section 4.1). 
Table 5: Summary table of the model results after pooling (consultation and surgery workstations)

\begin{tabular}{lcc}
\hline $\mathrm{i}$ & 1 & 2 \\
\hline$\frac{1}{\mu_{i}}$ & 246.90 & 995.87 \\
$C_{s_{i}}^{2}$ & 1.334 & 0.224 \\
$\frac{1}{\lambda_{i}}$ & 43.56 & 173.2 \\
$C_{a_{i}}^{2}$ & 0.996 & 1.075 \\
$A_{i}$ & 0.101 & 0.079 \\
$\rho_{i}$ & 0.944 & 0.958 \\
$E\left[W_{i}\right]$ (pooled) & 0.518 & 1.612 \\
$E\left[W_{i}\right]$ (non-pooled) & 4.523 & 12.47 \\
\hline
\end{tabular}

The benefits of pooling are clear. Without increasing capacity or altering any of the other system characteristics (except of course the pooling of capacity) we are able to reduce patient flow times at the consultation and surgery level by a factor of 8.73 and 7.74 respectively.

Unfortunately, it is often impossible to achieve such a high degree of pooling in a real life hospital system. One quickly runs into a number of limitations:

- Unique relation between patient and surgeon; patients will often refuse to consult another surgeon.

- Limited flexibility of resources; each surgeon has his own specialization. It is often impossible, even for surgeons at the same department, to pass on jobs. In other words, the flexibility of surgeons is limited.

- Resources often operate at different time instances; for pooling to take place surgeons need to operate at the same time instance. Due to busy schedules and other limitations, this is not always possible.

- Structural characteristics may further limit the practical applicability of pooling. For instance, if only two operating theatres are available, it is impossible to pool the capacity of the six surgeons at the surgery level. In other words, the bottleneck has shifted from the surgeons onto the number of available operating theatres.

Notwithstanding these constraints, it should be clear that even a small amount of pooling may yield significant reductions in patient flow time. Therefore the pooling of hospital resources is a worthwhile matter for further investigation.

\subsection{Finding the optimal number of patients in a service session}

The impact of absences at the start of a consultation or surgery session is discussed in Babes et al. (1991), Liu et al. (1998a), Liu and Liu (1998b) and Easton et al. (2005). There is a general agreement on the disruptive effect of absences on patient flow time. Easton et al. (2005) identify robust staffing, scheduling and recovery practices to minimize the effects of 
absences. Liu et al. (1998b) acknowledge the importance of consultation and surgery block size (i.e. the number of patients treated during a consultation session) and propose a what-if simulation approach in order to determine the best block size.

In fact, the relationship between block size and patient flow time is akin to the relationship between batch size and waiting time (in the presence of setups between batches in a manufacturing setting). As such the convex relationship first described by Karmarkar (1987) may also be observed here. In this view, Vandaele, Van Nieuwenhuyse and Cupers (2003b) determine the optimal size of patient groups queueing in front of a nuclear resonance scanner. We build on the model of Lambrecht and Vandaele (1996) in order to determine the optimal number of patients that receive treatment during a service session.

Two conflicting effects may be observed:

- The grouping effect; referring to the time required to assemble a batch of size $n$. The larger the batch size, the longer patients will have to wait before receiving service.

- The saturation effect; the smaller the batch size, the more service sessions are initiated, the larger the probability of having an absence of medical staff at the start of a service session.

We illustrate these effects in Figure 6.

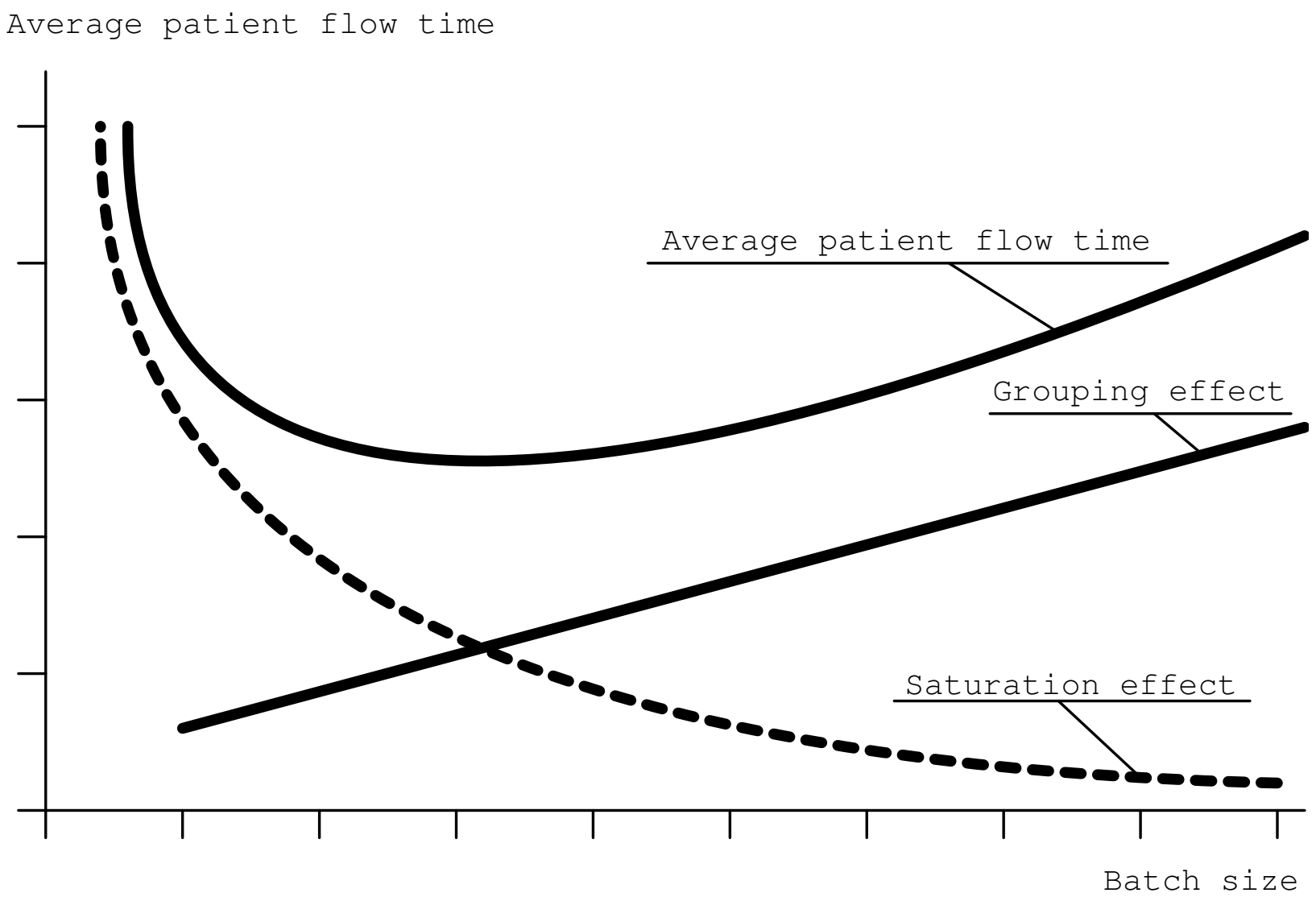

Figure 6: Convex relationship between average patient flow time and batch size 
The combination of both effects results in a convex relationship, which implies that there is an optimal group size minimizing average patient lead time. In what follows, we develop the mathematical model to address the batch size decision problem. The objective is to determine the batch size that minimizes the average patient lead time.

In this section we build on the third workstation discussed in the base case (other workstations at the consultation and surgery level may also be analyzed in a similar fashion). To maintain the transparency of the model, we omit the index $i$ referring to the original workstation used in this experiment. Other than the batching of patients, the dynamics of the workstation remain unchanged (as compared to previous sections).

Once sufficient patients are available, a batch (i.e. the equivalent of a service session workload) is created and is introduced into a queue (it is clear that this grouping does not imply that patients have to wait physically in the hospital). Whenever the server is idle, the batch as a whole receives service. After service, the batch is separated and patients resume their individual routings. A batch of patients is characterized by:

- a batch size $n$,

- a batch arrival rate $\lambda_{b}$,

- a coefficient of variation of the interarrival times of the batches $C_{a_{b}}^{2}$.

- a batch service rate $\mu_{b}$,

- a coefficient of variation of the service times of the batches $C_{s_{b}}^{2}$,

where

$$
\begin{aligned}
\lambda_{b} & =n \lambda, \\
C_{a_{b}}^{2} & =\frac{C_{a}^{2}}{n}, \\
\mu_{b} & =n \mu, \\
C_{s_{b}}^{2} & =\frac{C_{s}^{2}}{n}
\end{aligned}
$$

and $\lambda, C_{a}^{2}, \mu, C_{s}^{2}$ are the respective arrival rate, the squared coefficient of variation of the interarrival times, the service rate and the squared coefficient of variation of the service times of the individual patients visiting the third workstation.

The flow time of a patient in this system contains the following elements:

- The collection time; the time required until sufficient patients have arrived and a batch may be processed. The larger the batch size, the longer it takes to gather sufficient patients in order to perform a batch service.

- The waiting time of the batch itself; other batches (i.e. service sessions) may have to be serviced first.

- The absence time; prior to the service of a batch of patients, there exists a probability that the surgeon (or another crucial hospital resource) is absent. The batch of patients has to wait for the surgeon in order to receive service. This absence time can be considered as a setup time for the batch. 
- The actual processing of individual patients in the batch.

We visualize the flow time of a patient in Figure 7 .

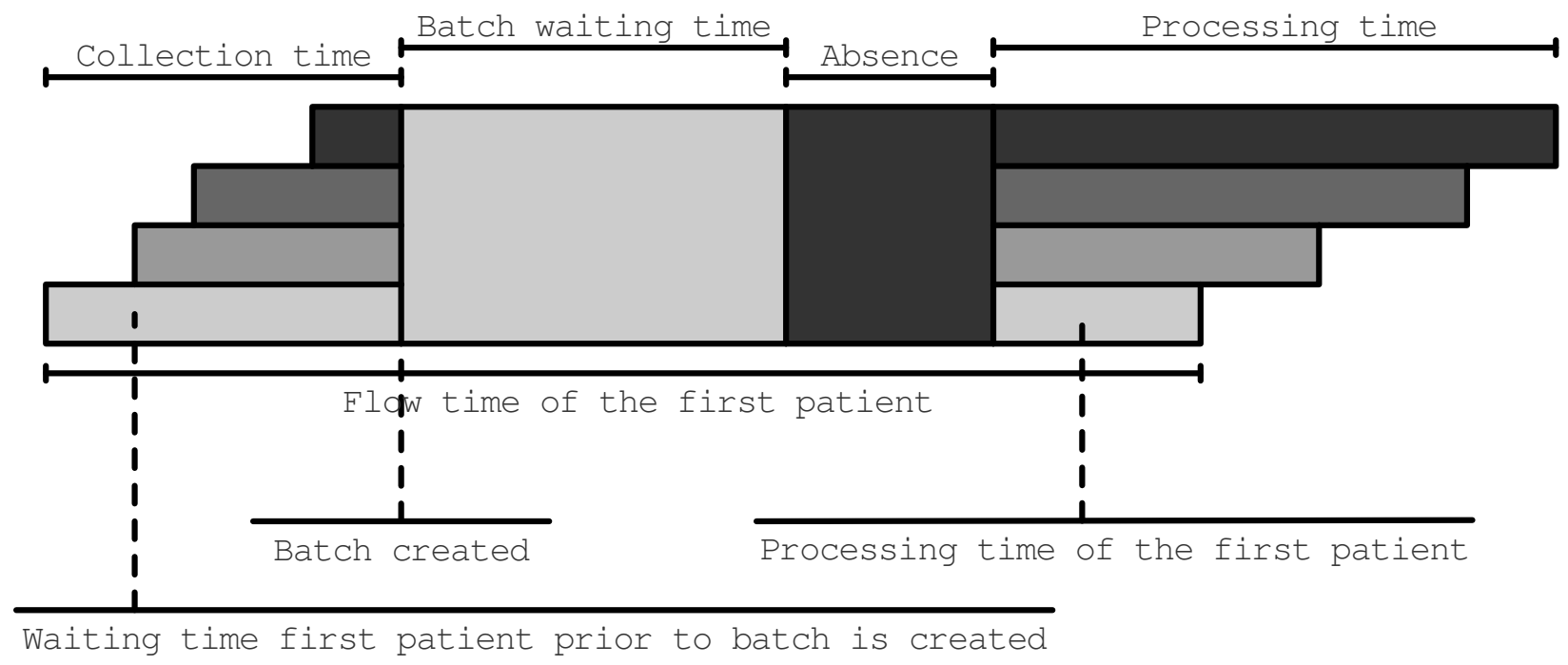

Figure 7: Visualization of the different phases of the batch flow time

The expected flow time of a single patient in the system can be expressed as (Lambrecht and Vandaele, 1996):

$$
E[W]=\frac{n-1}{2 \lambda}+E\left[W_{q}\right]+\frac{1}{\mu_{s}}+\frac{n+1}{2 \mu} .
$$

This flow time clearly consists of four building blocks. The first term corresponds to the average time a patient will have to wait until a group of size $n$ has been formed (i.e. the collection time). The term $E\left[W_{q}\right]$ stands for the average time that a batch of patients spends waiting in queue until the server becomes idle. We approximate $E\left[W_{q}\right]$ by means of the Kingman equation and obtain:

$$
E\left[W_{q}\right]=\left(\frac{C_{a_{b}}^{2}+C_{s_{b}}^{2}}{2}\right)\left(\frac{\rho}{1-\rho}\right) \frac{1}{\mu_{b}},
$$

where $\rho$ is the effective utilization rate at the third workstation and is given by (Lambrecht et al., 1996):

$$
\rho=\frac{n \lambda}{n \mu+\mu_{s}} .
$$

The third term corresponds to the absence time that is incurred at the start of a service session in which a batch of patients receives treatment. Both the second and third term are the same for all patients in the batch. The last term indicates how much time a patient spends on processing itself. At this point the model is complete and we can formally state our optimization problem:

$$
\begin{array}{ll}
\text { Minimize } E[W], & E[W]=\frac{n-1}{2 \lambda}+E\left[W_{q}\right]+\frac{1}{\mu_{s}}+\frac{n+1}{2 \mu}, \\
\text { s.t. } & \rho<1 \\
& n \geq 1
\end{array}
$$


When using the setting of the hospital department outlined in the previous sections, we are able to provide a numerical example. To maintain transparency, we select a single consultation workstation and assess different values of $n$ in order to obtain the optimal number of patients to be treated during a service session. A summary of the resulting figures is presented in Table 6 .

Table 6: Summary table of the model results featuring different batch sizes

\begin{tabular}{ccccc}
\hline$n$ & $\frac{1}{\mu_{b}}$ & $C_{s_{b}}^{2}$ & $\rho$ & $E[W]$ \\
\hline 3 & 82.063 & 0.2276 & 1.0787 & $\mathrm{NA}$ \\
4 & 99.418 & 0.1707 & 0.9802 & 27.460 \\
5 & 116.77 & 0.1365 & 0.9210 & 8.2226 \\
6 & 134.13 & 0.1138 & 0.8815 & 6.3769 \\
7 & 151.48 & 0.0975 & 0.8534 & 5.8782 \\
8 & 168.84 & 0.0853 & 0.8322 & 5.7761 \\
9 & 186.19 & 0.0758 & 0.8162 & 5.8441 \\
10 & 203.54 & 0.0683 & 0.8027 & 6.0004 \\
11 & 220.90 & 0.0621 & 0.7919 & 6.2086 \\
12 & 238.25 & 0.0569 & 0.7830 & 6.4497 \\
13 & 255.61 & 0.0525 & 0.7754 & 6.7132 \\
14 & 272.96 & 0.0488 & 0.7689 & 6.9924 \\
15 & 290.32 & 0.0455 & 0.7632 & 7.2831 \\
16 & 307.67 & 0.0427 & 0.7583 & 7.5826 \\
17 & 325.03 & 0.0402 & 0.7540 & 7.8888 \\
18 & 342.38 & 0.0379 & 0.7501 & 8.2004 \\
19 & 359.73 & 0.0359 & 0.7466 & 8.5162 \\
20 & 377.09 & 0.0341 & 0.7435 & 8.8355 \\
\hline
\end{tabular}

An illustration is provided in Figure 8. 


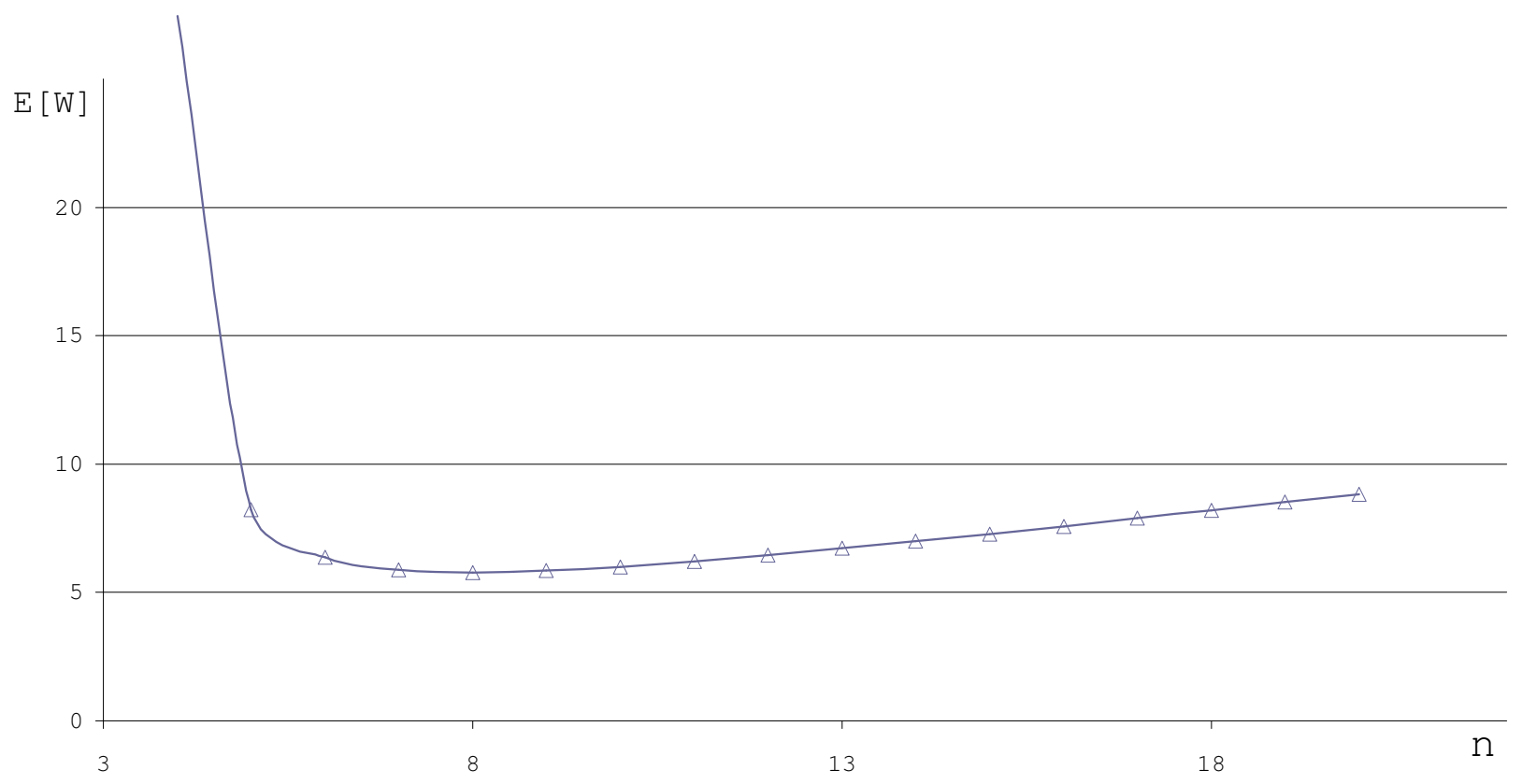

Figure 8: Finding the optimal number of patients

One can deduce that, for this particular workstation, the optimum is reached when treating 8 patients during each service session. More precisely, given a set of input parameters (absence probability, service and interarrival times, ...) we are able to determine the optimal number of patients to be treated during a service session.

\section{Conclusion}

In this article we discuss some of the features that differ when modeling healthcare queueing models on the one hand and traditional manufacturing models on the other hand. We show how to implement these features in a hospital queueing network. We used the parametric decomposition approach to assess performance measures at the hospital queueing network. In addition, we develop new expressions to model service outages that are typical in services in general and in healthcare in particular. The resulting queueing network is used to construct a numerical example and to illustrate a number of practical applications. First we demonstrate the detrimental effect of service interrupts on patient flow times. Next, the beneficial effect of pooling hospital resources is illustrated. Finally, we develop an optimization model that is able to determine the optimal number of patients treated during a single service session.

Notwithstanding these accomplishments, there is still room for improvement. More specifically, improvements may be made with respect to the modeling of time in queueing systems. Open problems include the modeling of time-dependent demand rates, increasing workload as waiting times increase (patients need to be monitored, receive care, ... ) , ... Moreover, given the inherent high degree of variability in service times, hospitals often use flexible working schedules that allow for overtime, variable server capacity and other deviations from the standard queueing model topology. Such deviations add to the complexity of the problem, making "time" a major modeling issue. 
doi:10.1007/978-1-4419-6472-4_18 • www.stefancreemers.be • $₫$ info@stefancreemers.be

\section{References}

[1] Askin RG (1993) Modeling and analysis of manufacturing systems. Wiley, New York

[2] Albin SL (1984) Approximating a point process by a renewal process, II: superposition arrival processes to queues. Operations Research 32:1133-1162

[3] Babes M, Sarma GV (1991) Out-patient queues at the Ibn-Rochd health center. Journal of the Operational Research Society 42:845-855

[4] Benjaafar S, Cooper WL (2005) On the benefits of pooling in production-inventory systems. Management Science 51:548-565

[5] Bitran GR, Tirupati D (1988) Multiproduct queueing networks with deterministic routing: decomposition approach and the notion of interference. Management Science 34:75100

[6] Buzacott JA, Shanthikumar JG (1985) Queueing Models of Dynamic Job Shops. Management Science 31:870-887

[7] Chisholm CD, Collison EK, Nelson DR, Cordell WH (2000) Emergency department workplace interruptions: are emergency physicians "interrupt-driven" and "multitasking". Academic Emergency Medicine 7:1239-1243

[8] Chisholm CD, Dornfeld AM, Nelson DR, Cordell WH (2001) Work interrupted: a comparison of workplace interruptions in emergency departments and primary care offices. Annals of Emergency Medicine 38:146-151

[9] Creemers S, Lambrecht MR (2007) Modeling a healthcare system as a queueing network: the case of a Belgian hospital. In: FBE publications: Research Reports and Discussion papers. Department of Decision Sciences \& Information Management, Research Center for Operations Management, Catholic University Leuven. Available via KULeuven. http://bib.kuleuven.be/ebib/wp.htm.Cited1Aug2008

[10] Doshi BT (1986) Queueing systems with vacations - a survey. Queueing Systems 1:29-66

[11] Dudewicz EJ, Mishra SN (1988) Modern mathematical statistics. John Wiley Sons, New York

[12] Easton FF, Goodale JC (2005) Schedule recovery: unplanned absences in service operations. Decision Sciences 36:459-488

[13] France DK, Levin S, Hemphill R, Chen K, Rickard D, Makowski R, Jones I, Aronsky D (2005) Emergency physicians' behaviors and workload in the precense of an electronic whiteboard. International Journal of Medical Informatics 74:827-837

[14] Gabow PA, Karkhanis A, Knight A, Dixon P, Eiser S, Albert RK (2006) Observations of residents' work activities for 24 consecutive hours: implications for workflow redesign. Academic Medecine 81:766-775 
[15] Green LV, Soares J (2007) Computing Time-Dependent Waiting Time Probabilities in $\mathrm{M}(\mathrm{t}) / \mathrm{M} / \mathrm{s}(\mathrm{t})$ Queueing Systems. M\&SOM Manufacturing \& Service Operations Management 9:54-61

[16] Hall RW (2006) Patient Flow: The New Queueing Theory for healthcare. OR/MS Today $23: 36-40$

[17] Hall RW, Belson D, Muralli P, Dessouky M (2006) Modeling patient flows through the healthcare system. In: Hall RW (ed) Patient flow: reducing delay in healthcare delivery, Springer Science, New York

[18] Haskose A, Kingsman BG, Worthington D (2002) Modelling flow and jobbing shops as a queueing network for workload control. International Journal of Production Economics $78: 271-285$

[19] Haque L, Armstrong MJ (2007) A survey of the machine interference problem. European Journal of Operational Research 179:469-482

[20] Harvey R, Jarrett PG, Peltekian KM (1994) Patterns of paging medical interns during night calls at two teaching hospitals. Canadian Medical Association Journal 151:307-311

[21] Hopp WJ, Spearman L (2000) Factory Physics. McGraw-Hill Higher Education, New York

[22] Jackson JR (1957) Network of waiting lines. Operations Research 5:518-521

[23] Jackson JR (1963) Jobshop-like queueing systems. Management Science 10:131-142

[24] Karmarkar US (1987) Lot sizes, lead times and in-process inventories. Management Science 33:409-418

[25] Khinchin AJ (1960) Mathematical Methods in the Theory of Queueing. Hafner, New York

[26] Kingman JFC (1962) On queues in heavy traffic. Journal of the Royal Statistical Society. Series B (Methodological) 24:383-392

[27] Lambrecht MR, Vandaele NJ (1996) A general approximation for the single product lot sizing model with queuing delays. European Journal of Operational Research 95:73-88

[28] Lambrecht MR, Ivens PL, Vandaele NJ (1998) ACLIPS: a capacity and lead time integrated procedure for scheduling. Management Science 44:1548-1561

[29] Lariviere MA, Van Mieghem JA (2004) Strategically seeking service: how competititon can generate Poisson arrivals. Manufacturing \& Service Operations Management 6:23-40

[30] Lehaney B, Clarke SA, Paul RJ (1999) A case of intervention in an outpatient department. Journal of the Operational Research Society 50:877-891 
[31] Liu L, Liu X (1998a) Block appointment systems for outpatient clinics with multiple doctors. The Journal of the Operational Research Society 49:1254-1259

[32] Liu L, Liu X (1998b) Dynamic and static job allocation for multi-server systems. IIE Transactions 30:845-854

[33] Marshall KT (1968) Some inequalities in queuing. Operations Research 16:651-668

[34] Palm C (1943) Intensittsschwankungen im Fernsprechverkehr. Ericsson Technics 44:1-89

[35] Roth A, Van Dierdonck R (1995) Hospital resource planning: concepts, feasibility and framework. Production and Operations Meanagement 4:2-29

[36] Shanthikumar JG, Buzacott JA (1981) Open queueing network models of dynamic job shops. International Journal of Production Research 19:255-266

[37] Sethuraman K, Tirupati D (2005) Evidence of bullwhip effect in healthcare sector: causes, consequences and cures. International Journal of Services and Operations Management 1:372-394

[38] Stecke KE, Aronson JE (1985) Review of operator/machine interference models. Journal of Production Research 23:129-151

[39] Suri R, Sanders JL, Kamath M (1993) Performance evaluation of production networks. In: Graves SC et al. (ed) Handbooks in Operations Research and Management Science, Vol. 4: Logistics of Production and Inventory, Elsevier Science Publishers, New York

[40] Takagi H (1988) Queueing analysis of polling models. ACM Computing Surveys 20:5-28

[41] Tian N, Zhang ZG (2006) Vacation queueing models. Springer Science, New York

[42] Tucker AL, Spear SJ (2006) Operational failures and interruptions in hospital nursing. Health Services Research 41:643-662

[43] van Merode GG, Groothuis S, Hasman A (2004) Enterprise resource planning for hospitals. International Journal of Medical Informatics 73:493-501

[44] Vandaele N, De Boeck L (2003a) Advanced resource planning. Robotics and Computer Integrated Manufacturing 19:211-218

[45] Vandaele N, Van Nieuwenhuyse I, Cupers S (2003b) Optimal grouping for a nuclear magnetic resonance scanner by means of an open queueing model. European Journal of Operational Research 151:181-192

[46] Vissers JMH, Bertrand JWM, De Vries G (2001) A framework for production control in health care organizations. Production Planning \& Control 12:591-604

[47] Volpp KGM, Grande D (2006) Residents' suggestions for reducing errors in teaching hospitals. The New England Journal of Medicine 348:851-855 
[48] Whitt W (1983) The queueing network analyzer. The Bell System Technical Journal 62:2779-2815

[49] Whitt W (1994) Towards better multi-class parametric-decomposition approximations for open queueing networks. Annals of Operations Research 48:221-248

[50] Whitt W (1995) Variability functions for parametric-decomposition approximations of queueing networks. Management science 41:1704-1715

[51] Whitt W (1999a) Decomposition approximations for time-dependent Markovian queueing networks. Operations Research Letters 24:97-103

[52] Whitt W (1999b) Partitioning Customers into Service Groups. Management Science 45:1579-1592

[53] Yu Y, Benjaafar S, Gerchak Y (2006) On service capacity pooling and cost sharing among independent firms. Department of Mechanical Engineering, University of Minnesota. Available via University of Minnesota. 